Case Report

\title{
A case of Fahr's syndrome with rare atypical presentation as hemiplegia.
}

\author{
Venkata Ravikumar Chepuri ${ }^{1} \&$ Himabindu Panta ${ }^{2}$ \\ ${ }^{1}$ Assistant Professor, ${ }^{2}$ Post Graduate, Department of M edicine, Rajiv Gandhi Institute of M edical Sciences [RIMS], \\ Kadapa, Andhra Pradesh, India. \\ Correspondence \\ Venkata Ravikumar Chepuri \\ Assistant Professor, Department of Medicine, Rajiv Gandhi Institute of M edical Sciences, \\ Kadapa - 516004, Andhrapradesh, India. \\ Mobile : +91 9346917014 E-mail : ravi657chepuri@gmail.com
}

\begin{abstract}
Calcification of basal ganglia or Fahr's disease is a rare disease characterized by bilateral and symmetrical intracranial deposition of calcium mainly in cerebral basal ganglia. Basal ganglia calcification secondary to endocrinological cause is known as Fahr's syndrome. M otor and neuropsychiatric symptoms are prominent features. We report a case presented with hemiplegia without any psychiatric symptoms. Radiological findings were suggestive of bilateral calcification of cerebellar hemispheres and basal ganglia. Parathyroid hormone levels were low with no significant findings in other investigations along with negative family history. Based on the radiological and biochemical findings the case was diagnosed as fahr's syndrome.
\end{abstract}

Keywords: Basal ganglia Calcification, Parathyroid hormone, Fahrs syndrome.

\section{Introduction}

Fahr's syndrome is characterized by basal ganglia calcification with clinical manifestations in the form of neuropsychiatric disorders, neurological symptoms, and cognitive symptoms. ${ }^{[1,2]}$ Basal ganglia calcification [BGC] can be idiopathic or secondary to genetic, metabolic, and endocrinological disorders. Idiopathic BGC is known as Fahr's disease(FD) and BGC secondary to endocrinological causes is known as Fahr's syndrome. ${ }^{[3]}$ Calcified deposits are made up of calcium carbonate and calcium phosphate, and are commonly located in the Basal Ganglia, Thalamus, Hippocampus, Cerebral cortex, Cerebellar subcortical white matter and Dentate Nucleus. ${ }^{[4]}$ Although calcifications can involve other structures, globus pallidus is most commonly involved. The movement disorders are the most common neurological symptoms and usually

\begin{tabular}{|c|}
\hline Access this article online \\
\hline Quick Response Code \\
\hline
\end{tabular}

manifest as spasticity, gait disorder, speech i $\mathrm{m} \mathrm{p}$ a i $\mathrm{rm}$ e $\mathrm{nt}$, parkinsonism, chorea, tremor, dystonia, and myoclonus. ${ }^{[5]}$ About $40 \%$ of patients with basal ganglia calcification may present initially with psychiatric features. ${ }^{[6]}$ There is no reliable correlation between age, extent of calcium deposits in the brain, and neurological deficit. Progressive neurological deterioration generally results in disability and death. ${ }^{[7]}$ In adult onset Fahr's disease, calcium deposition generally begins in the 3rd decade of life, with neurological deterioration two decades later. ${ }^{[8]}$ We are reporting this case to document the rare and atypical presentation of fahr's syndrome as there were only a very few case reports in the literature.

\section{Case History}

A 50 years old male, who is a farmer, presented with sudden onset of weakness of right upper and lower limb which was not progressing, followed by loss of consciousness for a period of $5 \mathrm{~min}$, while he was working in his field. There was no history of seizures, headache and vomiting. There was no history of fever or head injury and no history of cerebrovascular accident [cva] or any other illness in the past. He was diabetic and was on regular treatment with oral hypoglycaemic agents. He was not a known smoker, non-alcoholic and there was no exposure to toxic substances. There was no significant family history. On physical examination he was drowsy but arousable. His 
Vitals were stable. Neurological examination revealed right sided hemiplegia, no sensory deficit, deep tendon reflexes were brisk, plantar reflexwas bilateral mute. There were no signs of meningeal irritation and cerebellar dysfunction. So the case was diagnosed as acute cva and it was managed accordingly.

Base line laboratory investigations were normal. Serum electrolytes and liver and renal function tests were normal. Electrocardiogram [ECG], 2DEchocardiography and neck vessel Doppler were normal. His viral screening was negative. Computerised Tomography scan [CT] of brain revealed bilateral symmetrical dense calcifications in cerebellar hemispheres [Figure: 1] and subtle calcifications in bilateral basal ganglia [Figure: 2]. After the CT scan further investigation were done Serum calcium, phosphorus, urine calcium was normal. But Parathyroid hormone levels were low. Based on the investigations, patient was diagnosed as Fahr's syndrome probably due to hypoparathyroidism.

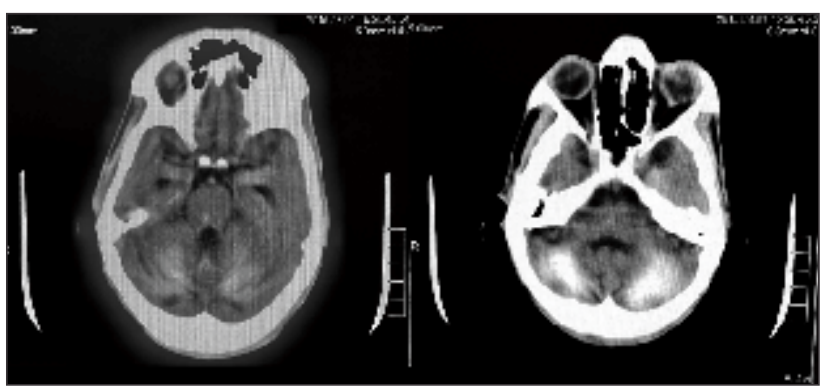

Figure 1 : Plain CTscan of brain showing bilateral symmetrical dense calcifications in cerebellar hemispheres.

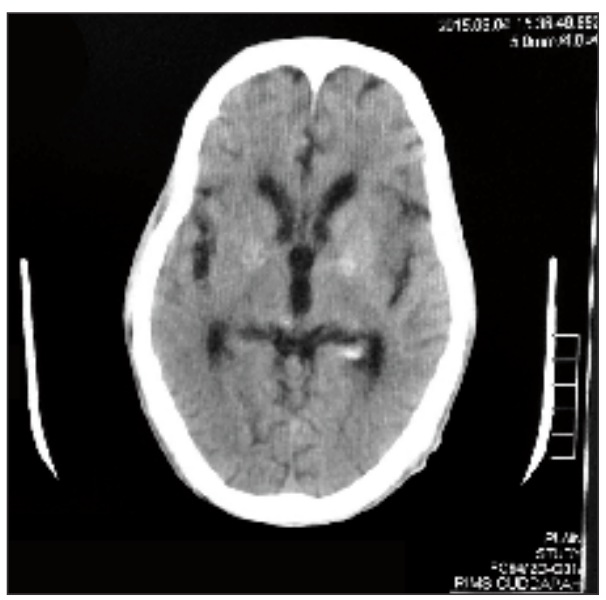

Figure 2: Plain CTscan of brain showing subtle calcifications in bilateral basal ganglia

\section{Discussion}

Fahr's syndrome is a rare disease. Fahr's syndrome is mostly associated with a disorder of calcium and phosphate metabolism, especially due to hypoparathyroidism. but can also be due to other different etiologies, including infectious, metabolic, and genetic diseases. ${ }^{[7]}$ The disease usually manifests itself in the 3rd to 5 th decade of life, but may appear in childhood or later in life. ${ }^{[8]}$ The exact prevalence of Fahr's syndrome is not known but intracranial calcifications can be detected incidentally in up to $0.3-1.2 \%$ of plain CT examinations of brain. ${ }^{[9]}$ Although bilateral and symmetric basal ganglia calcification is known to be associated with multiple medical conditions, the exact etiology is still unknown. ${ }^{[10]}$ $M$ any of these conditions involve the basal ganglia only or predominantly. The most common neurological manifestations include headache, seizures and movement disorders. Other specific manifestations include gait disturbances, dystonia, paresis, speech alterations, dementia, parkinsonism, tremors, chorea, etc. ${ }^{[10,11]}$ When latent Tetany and myopathic changes occur with changes in somatosensory, visual and brain stem auditory responses, then parathyroid dysfunction, mitochondrial disease or other disease associated with brain calcification may be considered. It is differentiated from calcified angiomas, infections and Addision's disease by its severity and characteristic distribution. ${ }^{[12]}$ Reduced blood flow to calcified regions correlates with clinical signs. ${ }^{[7]}$ Psychiatric manifestations most commonly include cognitive and psychotic disorders. ${ }^{[10,13]}$ which were not observed in our case. Our case is unique, as our patient presented with hemiplegia but no psychiatric manifestations before or after the presentation and no abnormality in calcium and phosphate levels, but parathyroid level was low. We believe that low parathyroid level may be the cause for BGC. These findings were similar to the case repoted by Faye.AD et al, a case of psychosis due to Fahr's syndrome with low parathyroid hormone but normal calcium and phosphorus. $^{[7]}$

\section{M olecular genetics}

Fahr's disease is most commonly transmitted as an 
Autosomal Dominant trait. But it may also be passed on as an autosomal recessive trait or it may occur sporadically. A Locus at $14 q$ (IBGC1) has been suggested to be involved commonly. A second locus has been identified on chromosome 8 and a third on chromosome 2. A loss of function mutation in the gene encoding type III sodium dependent phosphate transporter 2(SLC20A2) located on chromosome 8 has also been reported to form the genetic basis of this disease. ${ }^{[12]}$

Laboratory examinations should include tests for blood calcium and parathormone which in addition to the other routine blood tests will help in differentiating idiopathic Fahr's syndrome (unremarkable laboratory test results) from secondary cases especially due to hypoparathyroidism. Plain CT brain will demonstrate the presence and extent of parenchymal calcification. In our case there were bilateral symmetrical dense cerebellar calcification and subtle basal ganglia calcification was seen. The treatment of Fahr's syndrome is directed to the identifiable cause especially hypoparathyroidism. In other

\section{References}

1. Miller R. A Theory of the basal ganglia and their disorders. Boca Raton: CRCPress, Taylor \& Francis;2008.

2. Taxer F, Haller R, Konig P.Clinical early symptoms and CT findings in Fahr syndrome.Nervenarzt 1986;57:583-88.

3. Ghormode D, Maheshwari U, Kate N, Grover S.Fahr's disease and psychiatric syndromes: A case series. Ind Psychiatr J 2011;20:136-8.

4. Saleem et al.Fahr's syndrome.Literature review of current evidence.Orphanet Journal of Rare Diseases2013;8:156.

5. Lauterbach EC, Cummings JL, Duffy J, Coffey CE, Kaufer D, Lovell M, et al. Neuropsychiatric correlates and treatment of lenticulostriatal diseases: A review of the literature and overview of research opportunities in Huntington's, Wilson's, and Fahr's diseases. A report of the ANPA Committee on Research. American Neuropsychiatric Association.J Neuropsychiatry Clin Neurosci 1998;10:249-66.

6. König P. Psychopathological alterations in cases of symmetrical basal ganglia sclerosis. Biol Psychiatry 1989;25:459-68.

7. Faye AD, Gawande S, Tadke R, Kirpekar VC, Bhave SH. A case of psychosis due to Fahr's syndrome and response to behavioral disturbances with risperidone and oxcarbazepine. Indian J Psychiatry 2014;56:188-90 cases, symptomatic or conservative therapy with clinical follow-up is the rule. Prognosis is variable, cannot be predicted and is unrelated to the extent of calcification. ${ }^{[14]}$

\section{Conclusion}

Though farh's syndrome is a rare incidental finding, routine biochemical investigations should always be performed to rule out metabolic causes. All patients with incidentally detected calcifications in basal ganglia and in other areas of brain should be subjected to thorough neuropsychiatric examination and biochemical tests. Whenever possible the associated conditions should be identified and managed accordingly. Currently, there is no cure for Fahr's syndrome, or a standard course of treatment. The available treatment is directed toward symptomatic control and treatment for cause of calcification. ${ }^{[7]}$ Brain CT scan serves as a presymptomatic test in at risk individual but it's not useful for predicting age of onset, severity, type of symptoms or rate of progression in an asymptomatic individual. Individuals with a positive test result need arrangements for long-term follow-up and evaluations. ${ }^{[2]}$

8. Manyam BV, Bhatt MH, Moore WD, Devleschoward $A B$, Anderson DR, Calne DB. Bilateral striopallidodentate calcinosis: Cerebrospinal fluid, imaging, and electrophysiological studies. Ann Neurol 1992;31:379-84.

9. Fenelon G, Gray F, Paillard F, Thibierge M, Mahieux F, Guillani A. A prospective study of patients with CT detected pallidal calcifications.J NeurolNeurosurgPsychiatry 1993;56:622-625.

10. Shigeyuki 0 . Imaging of bilateral striopallidodentate calcinosis.Clin Nucl Med 2002;27:721-724

11. Modrego PJ, Mojonero J, Serrano M, Fayed N. Fahr's syndrome presenting with

pure and progressive presenile dementia. NeurolSci2005;26:376-379.

12. Saleem S, Aslam HM, Anwar M, et al. Fahr's syndrome: literature review of current evidence. Orphanet Journal of Rare Diseases. 2013;8:156

13. Gulsun M, Baykiz AF, Kabatas S, Belli H. Fahr's Syndrome-three casespresenting with psychiatric signs. EurJ Gen M ed 2006;3:35-40.

14. Senoglu M, Tuncel D, Orhan FO, Yuksel Z, Gokce M. Fahr's syndrome: a report of two cases. Firat Tip Dergisi 2007;12:70-72. 